\section{Nanominerals and biochar as additives in the composting of agricultural wastes effects on GHG emissions, composition and biodegradability of end-products on grassland soilso Medina, J.ab, Calabi-Floody, M.c, Panettieri, M.d, Cornejo, P.a, Borie, F.af, Knicker, Hd.}

\author{
a Centro de Investigación en Micorrizas y Sustentabilidad Agroambiental (CIMYSA), Scientific and Technological Bioresources Nucleus-BIOREN, Universidad de La Frontera, Temuco, Chile. \\ Instituto de Ciencias Agronómicas y Veterinarias, Universidad de O'Higgins, San Fernando, Chile. \\ Resources Biotechnology, Scientific and Biotechnological Bioresource Nucleus, BIOREN-UFRO,
$\mathrm{d}$ Instituto de Recursos Naturales y Agrobiología de Sevilla (IRNAS-CSIC), Seville, Spain. \\ 'Facultad de Recursos Naturales. Universidad Católica de Tanca, Casilla Postal 58-D Temuco, Chile. \\ "corresponding author: jorge.medina@ Qufrontera.cl; jorge.medinaeuoh.cl
}

\section{Introduction}

The utilization of additives is a strategy commonly used in composting operations to enhance the physicochemical properties and optimize the process. However, little is known about the impact of nanominerals, biochar and their combination during composting. The objective of this research was to evaluate the effects of iron oxide/halloysite nanominerals and oat hull-biochar as additives in the physicochemical properties of an aerobic composting process, the emission of greenhouse gases (GHG) and the composition of end-products. a)

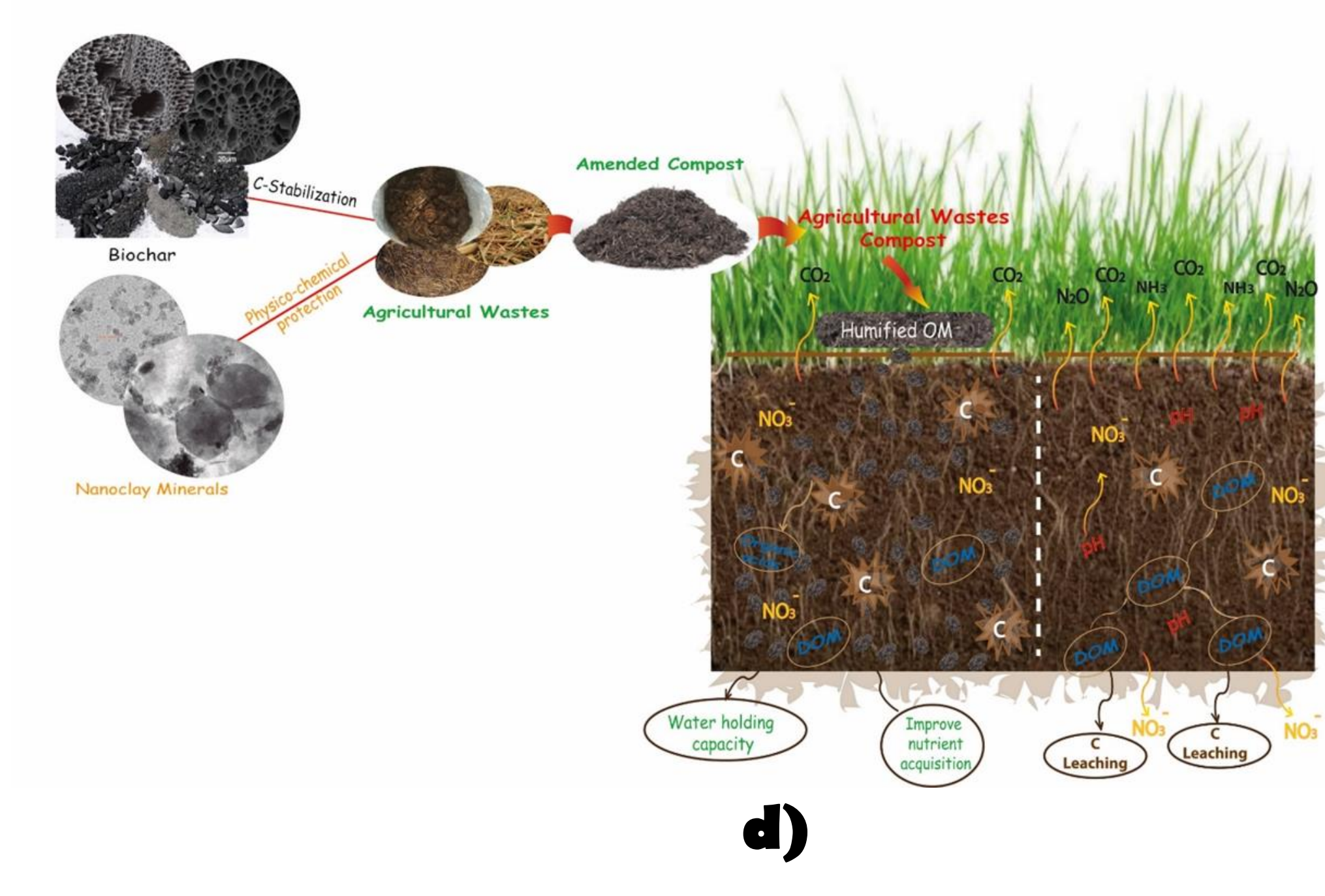

b)

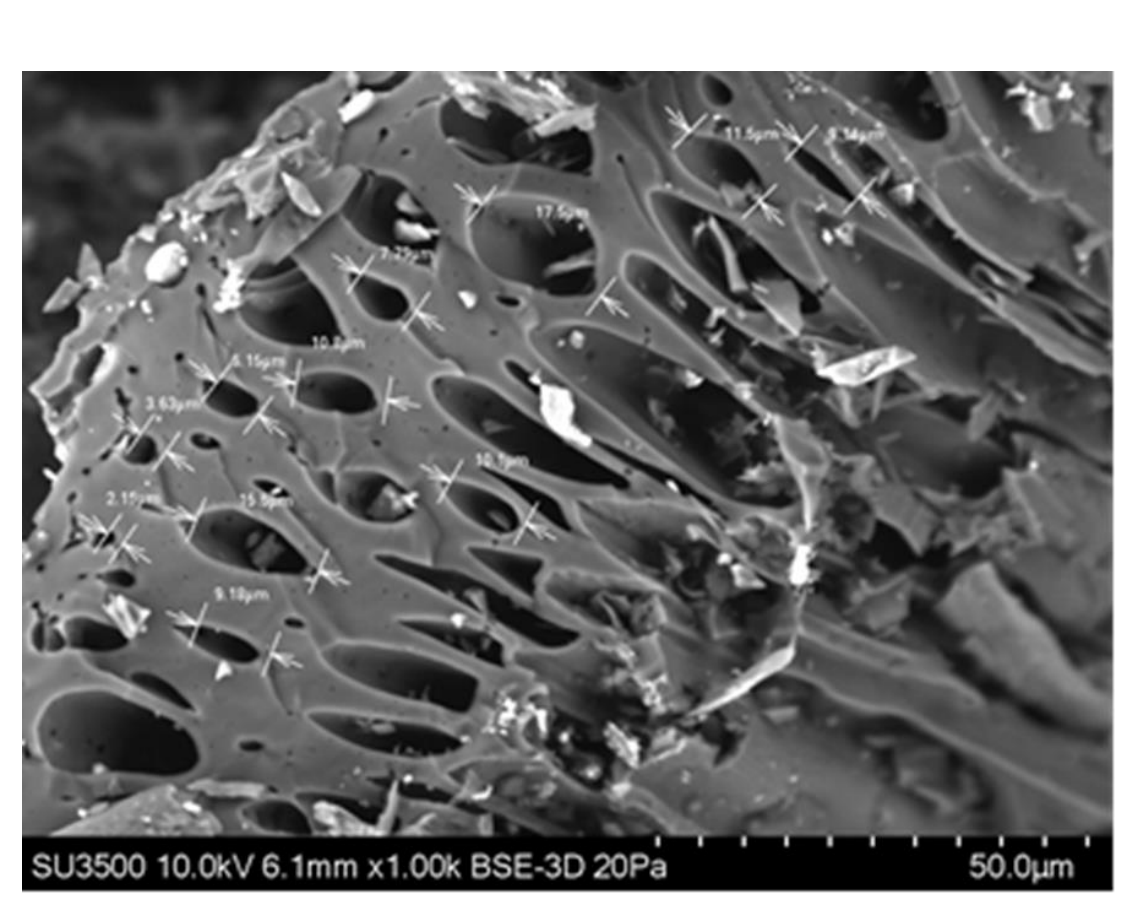

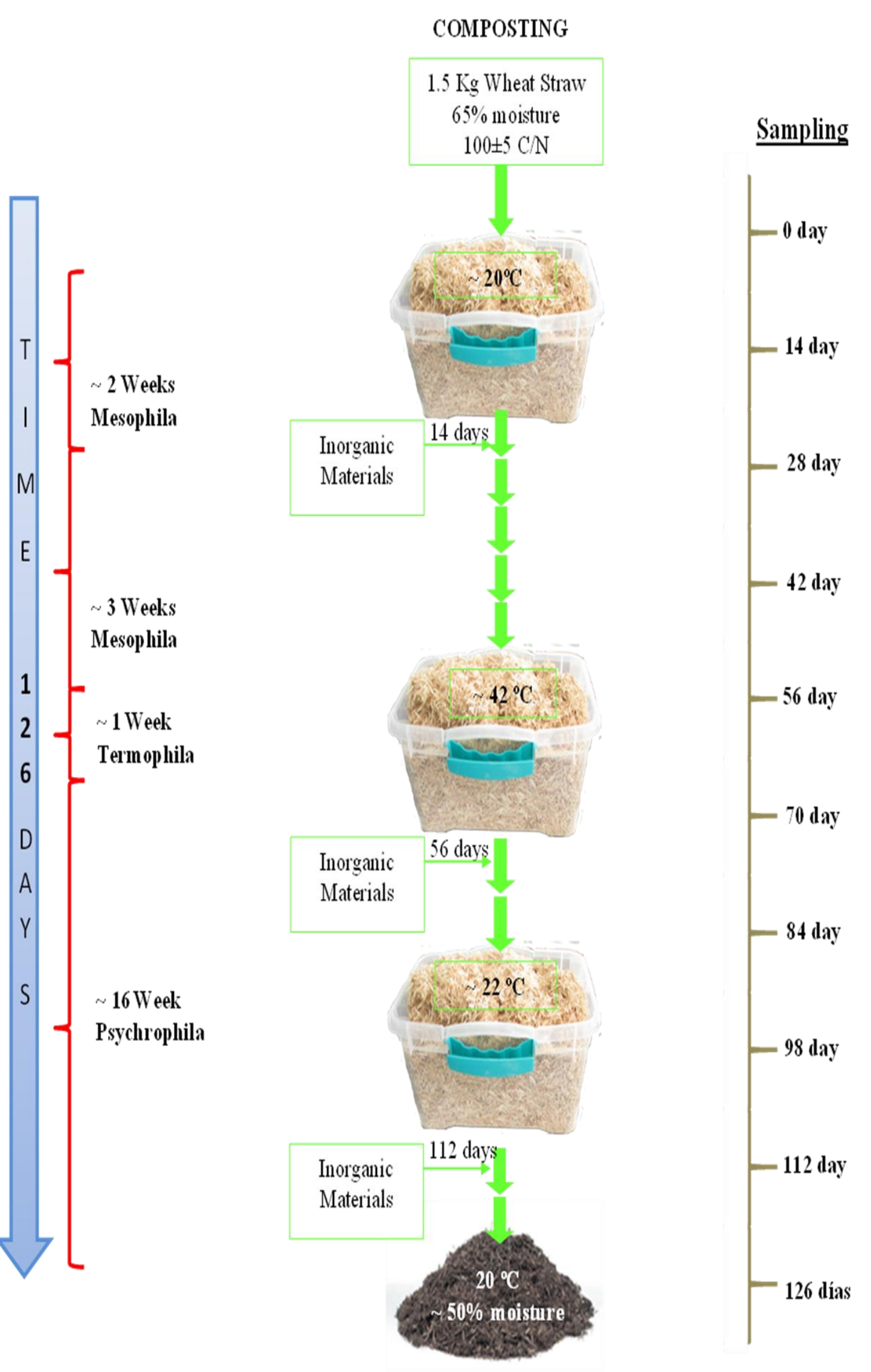

Figure 1. a) Graphical abstract; b) schematic representation of composting process; c) GHG analysis and d) biochar.

\section{Materials and Methods}

- Wheat straw, lupine and beef manure composting mixture (C/N: $\sim 25)$.

- Iron oxide (Fe) or halloysite ( $\mathrm{Ha})$ nanoparticles ( $2 \% \mathrm{w} / \mathrm{w})$, oat-biochar (B) $(7 \% \mathrm{w} / \mathrm{w})$ and their combination $(\mathrm{BFe}, \mathrm{BHa})$ were applied as additives.

- Physicochemical properties, the emissions of $\mathrm{CO}_{2}-\mathrm{CH}_{4}$ were determined during the process (128 days) (Sánchez-Monedero et al., 2010).

- The end-products characterized by nuclear magnetic resonance $\left({ }^{13} \mathrm{CNMR}\right)$.

- Respiration experiment (Respicond) combined with $\delta^{13} \mathrm{C}$ isotopic analysis was conducted.

\section{Conclusion}

These results suggest that the addition of halloysite and biochar to composting operations have significant effects on $\mathrm{C}$ stabilization and biodegradability of compost in grassland soils, that is relevant in the production of $C$ sequestrant amendments.

\section{Acknowledgements}

FONDECYT N ${ }^{\circ} 3170677$

\section{References}

Sánchez-Monedero, M., Serramiá, N., García-Ortiz Civantos, C., Fernandez, A

Roig, A. 2010. Greenhouse gas emissions during composting of two-phase olive mill wastes with different agroindustrial by-products. Chemosphere 81, 18-25

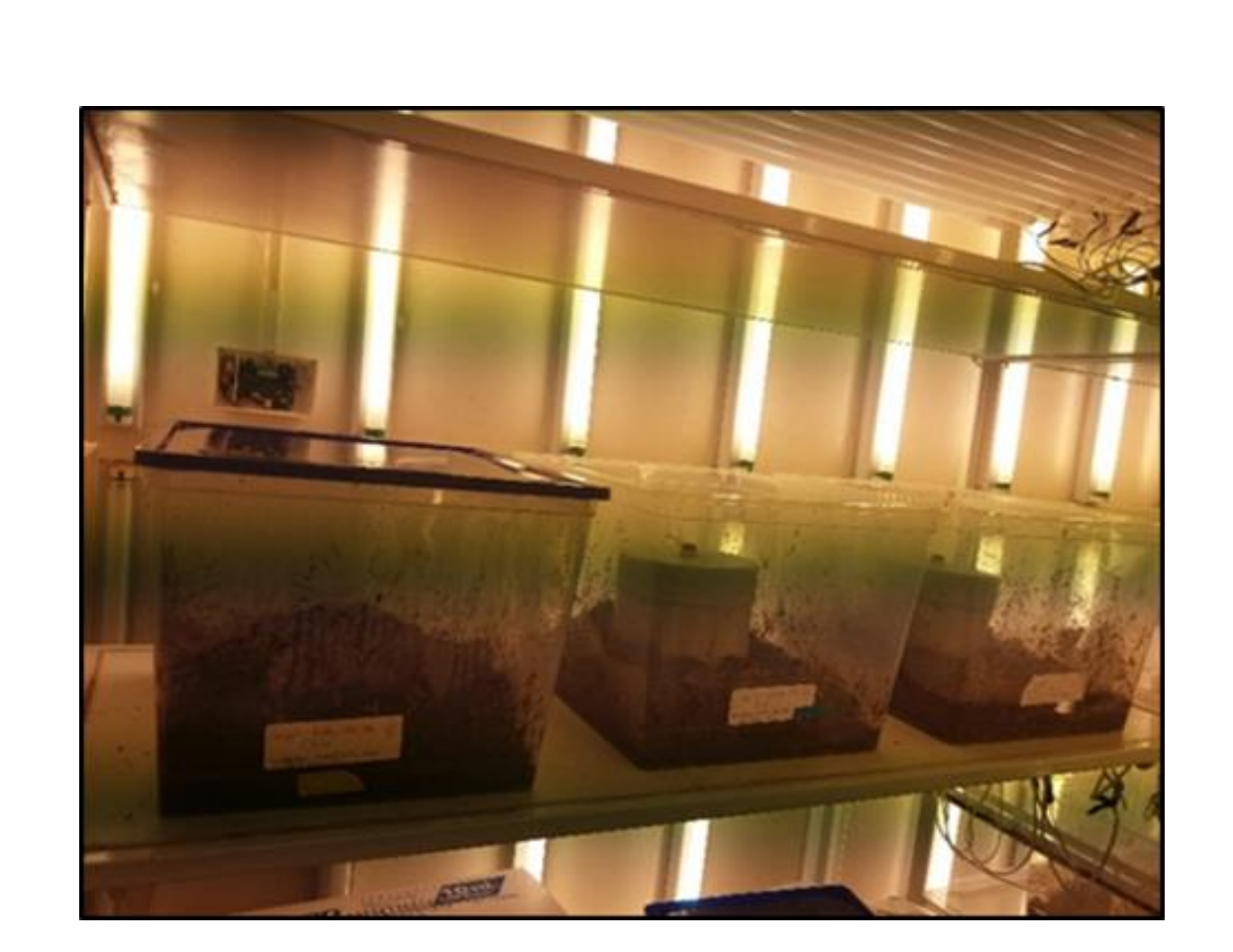

\section{Result and Discussion}

A decrease of final $\mathrm{C} / \mathrm{N}$ ratio was observed in all treatments that was lower in $\mathrm{B}$ treated compost. Nitrate concentration increased as composting progressed, and compost supplied with Ha showed the higher final content of $\mathrm{NO}_{3}(5800 \mathrm{mg}$ $\mathrm{kg}^{-1}$ ) and $\mathrm{NH}_{4}\left(220 \mathrm{mg} \mathrm{kg}^{-1}\right)$. The addition of $\mathrm{B}$ significantly decreased the mean emission of both $\mathrm{CO}_{2}\left(\sim 400 \mathrm{~g} \mathrm{CO}_{2} \mathrm{~m}^{2} \mathrm{~d}^{-1}\right)$ and $\mathrm{CH}_{4}\left(\sim 4.5 \mathrm{~g} \mathrm{CH}_{4} \mathrm{~m}^{2} \mathrm{~d}^{-1}\right)$. Nanominerals significantly decreased the final E4/E6 ratio $(<6)$ and the addition of $B$ increased the aromaticity (twice), the alkyl-C/O alkyl-C ratio and the hydrophobicity which are parameters associated to stabilized end-products. In soil, the incorporation of additives reduced the loss of $C$ ( $<5 \%$ after 60 days of incubation). Treatments supplied with $\mathrm{B}$ and $\mathrm{Ha}$ showed a higher mean residence time ( 8 and 5 years respectively) than compost without additives.

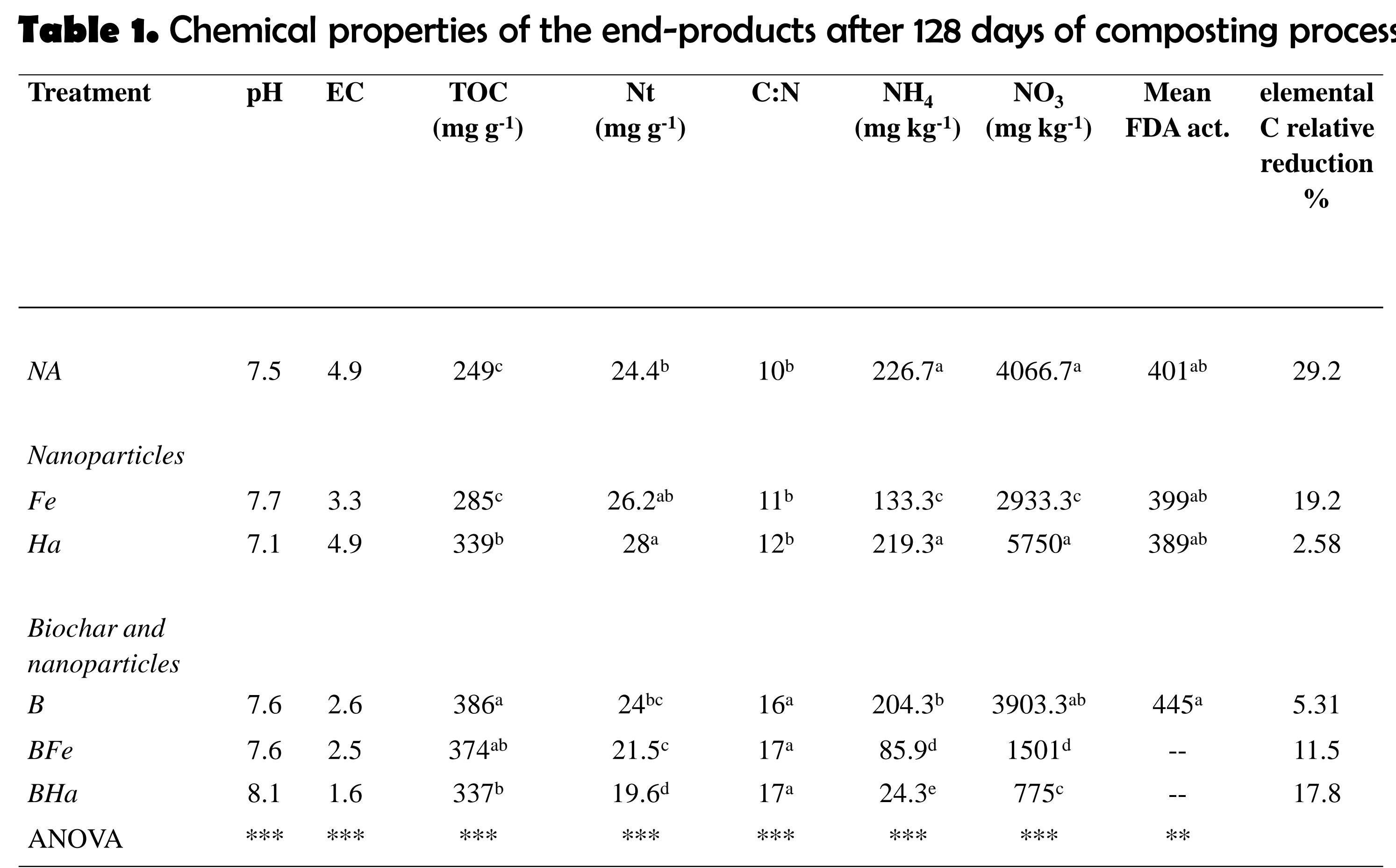

a)

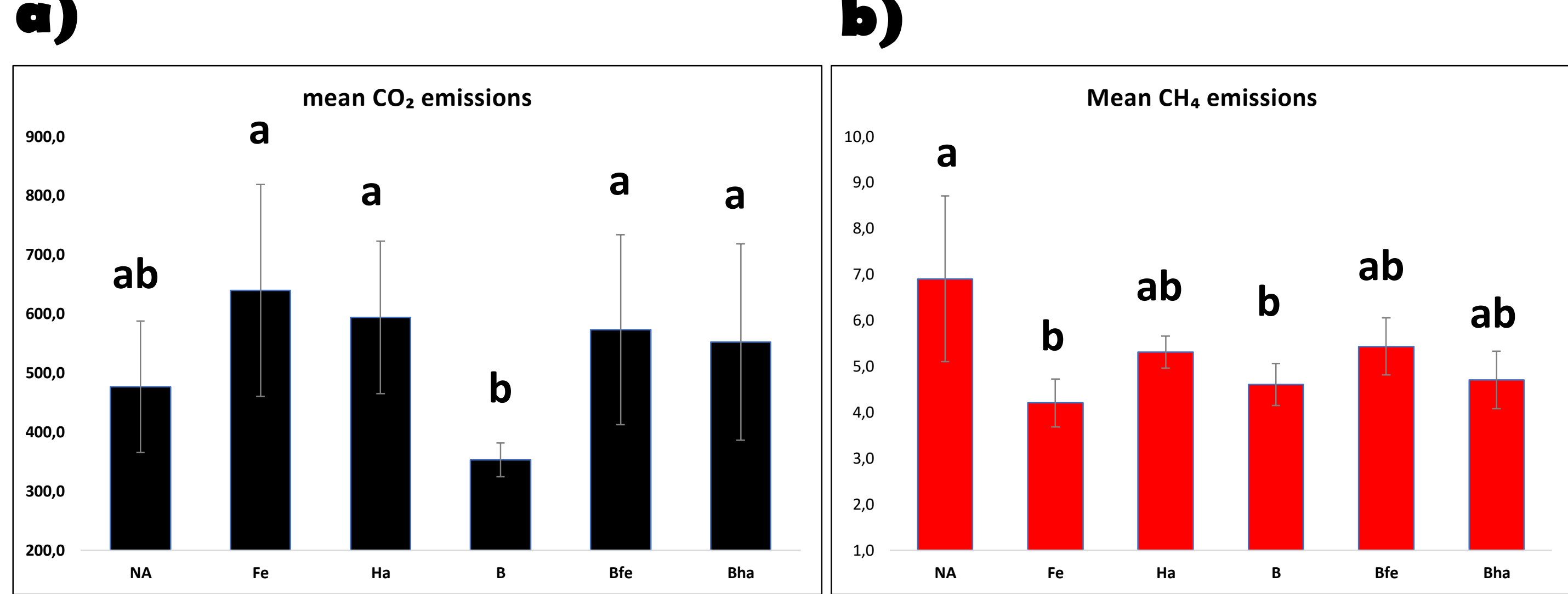

Figure 3. Mean a) $\mathrm{CO}_{2}$ and b) $\mathrm{CH}_{4}$ emissions from composting treatments

Table 2. Degradation constants, $C$ losses and MRT of compost in grassland soil.

\begin{tabular}{|c|c|c|c|c|c|c|c|c|}
\hline Treatment & 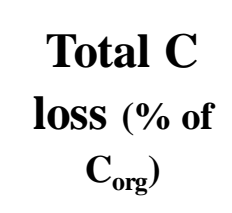 & 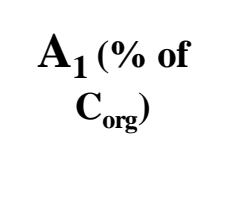 & $\mathbf{K}_{1}$ (year - 'I) & $\mathrm{MRT}_{1}$ (years) & $\begin{array}{l}\mathrm{A}_{2}\left(\mathrm{C}_{\mathrm{O}} \text { of }\right. \\
\mathrm{Cog}^{2}\end{array}$ & $\mathbf{K}_{2}($ vear -1$)$ & $\mathrm{MRT}_{2}$ (years) & $t_{1 / 2 \text { (gears) }}$ \\
\hline Soil & $2.8 \pm 0.3$ & $1.25 \pm 0.3$ & $64.2 \pm 2.7$ & $0.0156 \pm 0.00$ & $98.7 \pm 0.3$ & $0.104 \pm 0.004$ & $9.2 \pm 0.4$ & $6.4 \pm 0.3$ \\
\hline No additives & $4.7 \pm 0.6$ & $1.7 \pm 0.3$ & $82.9 \pm 11.0$ & $0.0122 \pm 0.00$ & 98.110 .4 & $0.225 \pm 0.025$ & $4.5 \pm 0.5$ & $3.1 \pm 0.4$ \\
\hline Iron oxide & $4.6 \pm 0.3$ & $1.9 \pm 0.2$ & $124.9 \pm 30$ & $0.0084 \pm 0.00$ & 98. 000.4 & $0.205 \pm 0.034$ & $5.0 \pm 0.8$ & $3.4 \pm 0.6$ \\
\hline Halloysite & $3.3 \pm 0.3$ & $1.0 \pm 0.5$ & $148.8 \pm 5.1$ & $0.0172 \pm 0.00$ & $99.0 \pm 0.5$ & $0.185 \pm 0.010$ & $5.4 \pm 0.1$ & $3.7 \pm 0.1$ \\
\hline Biochar & $3.8 \pm 0.6$ & $2.1 \pm 0.5$ & $36.2+6.0$ & $0.0281 \pm 0.00$ & $97.8 \pm 0.6$ & $0.132+0.012$ & $7.6 \pm 0.7$ & $5.3 \pm 0.5$ \\
\hline Biochar $+\mathrm{Fe}$ & $5.0 \pm 0.2$ & $0.8 \pm 0.1$ & $137.5 \pm 32.7$ & $0.0070 \pm 0.001$ & 99.220.1 & $0.300 \pm 0.03$ & $3.2 \pm 0.3$ & $2.2 \pm 0.2$ \\
\hline Biochar+Ha & $5.2 \pm 0.4$ & $1.0 \pm 0.1$ & $154.47 \pm 4.8$ & $0.0065 \pm 0.00$ & 99.00.1 & $0.319 \pm 0.00$ & $3.2 \pm 0.4$ & $2.2 \pm 0.3$ \\
\hline
\end{tabular}

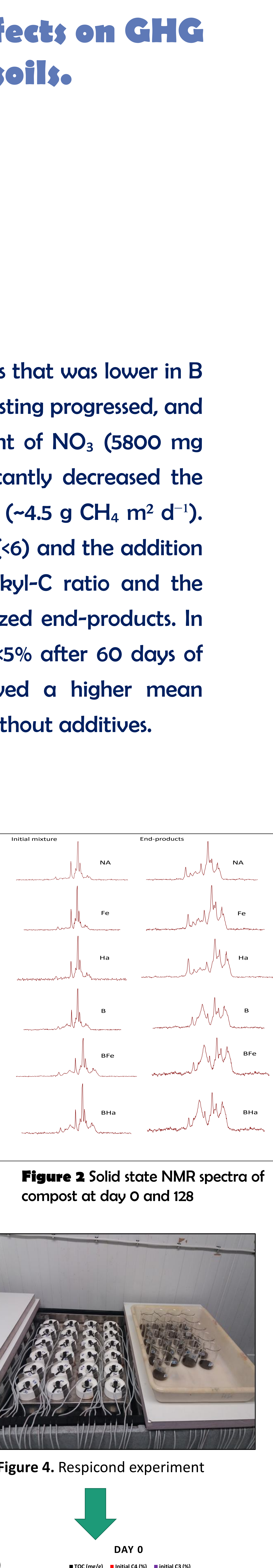

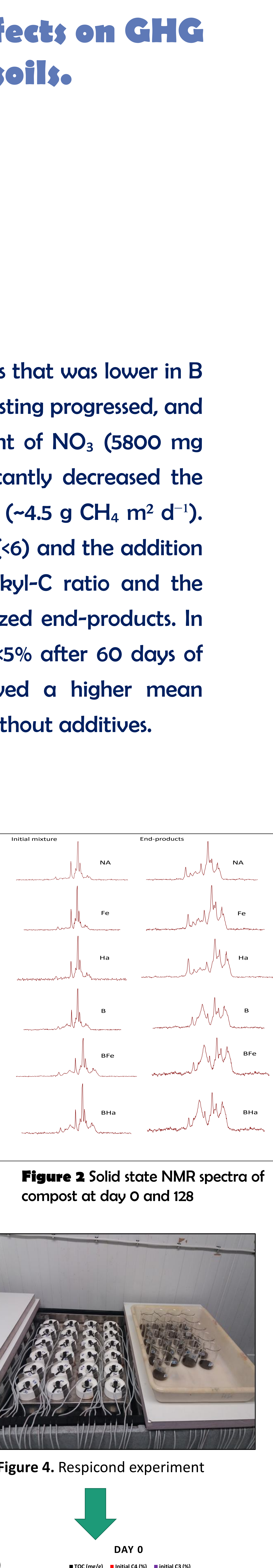

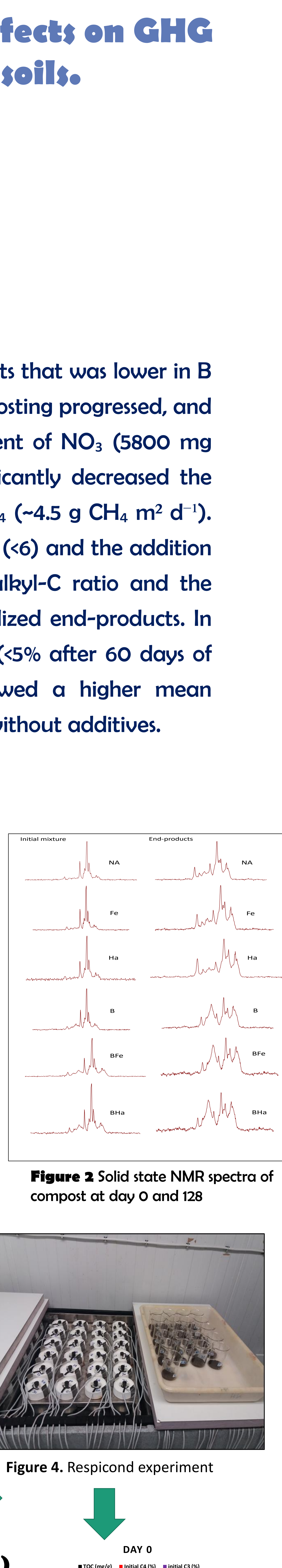

a)
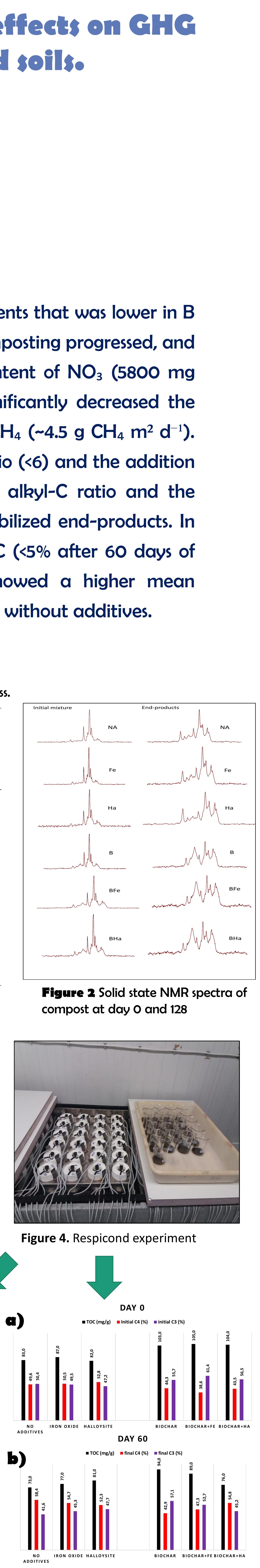

Figure 5. Fraction of $O C$ derived from amendments $(\delta 13) a)$ at day $O$ and $b$ ) day 60 of soil-compost incubations 